\title{
Perfect Light Absorption in Graphene by Two Unpatterned Dielectric Layers and Potential Applications
}

\author{
Jinfeng Zhu, ${ }^{1 *}$ Chawei Li, ${ }^{1}$ Jun-Yu Ou ${ }^{2}$ and Qing Huo Liu ${ }^{3}$ \\ ${ }^{1}$ Department of Electronic Science, Xiamen University, Xiamen, 361005, \\ China \\ ${ }^{2}$ Optoelectronics Research Centre and Centre for Photonic Metamaterials, \\ University of Southampton, Highfield, Southampton, SO17 1BJ, UK \\ ${ }^{3}$ Department of Electrical and Computer Engineering, Duke University, \\ Durham, NC 27708, USA
}

\begin{abstract}
In the spectral range from ultraviolet to near infrared, graphene lacks the capability to support plasmon polaritons, and has low optical absorptivity for applications due to its extremely small thickness. Many photonic structures based on sophisticated nanofabrication or metal plasmonics have been adopted to conquer this limitation, but they suffer from high expenses or metal parasitic losses. Here, a single-channel coherent perfect absorber simply based on two unpatterned dielectric layers is proposed to reach $\sim 100 \%$ light absorption in monolayer and few-layer graphene. The schemes for narrowband and broadband perfect absorption in graphene are systematically demonstrated, and their potential applications on fibre-integrated narrowband perfect absorbers, high-performance optical sensors, electric-optic modulators and broadband perfect absorbers are also investigated. Our research provides a simple and costeffective method to completely trap the light from ultraviolet to near infrared in a subnanometre scale for a lot of high-performance photonic and optoelectronic devices based on graphene and potentially other 2D materials.
\end{abstract}

\section{Introduction}

The family of 2D materials has generated significant interest in the field of photonics and optoelectronics owing to their novel properties for light-matter interactions, which is totally different from those of their $3 \mathrm{D}$ counterparts $[1,2]$. Along with the recent emergence of 2D lateral heterostructures [3,4], the study of light trapping in subnanometre 2D materials has become more and more significant in photo-detection, photovoltaics, photoluminescence, Raman microscopy, optical sensing and optoelectronic modulation [5-8]. As the most popular representative of 2D materials, graphene has attracted tremendous attention from the optical communities of research *Corresponding author. E-mail: nanoantenna@ hotmail.com 
and industry, and has been systematically studied for the spectral range from ultraviolet (UV) to terahertz $[9,10]$. In the spectral range from mid-infrared to terahertz, graphene is capable to support effects of plasmon polaritons, which can lead to strong light-matter interaction by a plasmonic coupling [11]. In contrast, for the range from UV to nearinfrared (NIR), intrinsic graphene acts as a lossy conductive surface, and suffers from low optical absorption (only $2.3 \%$ for normal incident visible-NIR light) due to an atomic layer structure with an extremely small thickness [12]. In order to enhance the optical absorption in graphene from UV to NIR, plentiful efforts have been made on combining graphene with various photonic structures, such as dielectric waveguides, photonic crystals, plasmonic metamaterials and integrated microcavities [13-16]. However, most of these methods need a sophisticated fabrication process with subwavelength patterning inside or outside graphene layers. Such a fabrication process will not only increase the manufacture complexity and cost, but also introduce possible fatal degradation of the electronic and photonic performance of graphene, because as a 2D material graphene is usually sub-nanometre thin and has the band structure very sensitive to atom-scale damage and environmental contaminant. Particularly, for UV applications, precise fabrication of nanoscale features on graphene might be much more difficult due to patterning with smaller subwavelength sizes. Previous efforts have aimed to achieve high optical absorption in graphene by using an unpatterned graphene/dielectric/metal structure with polarization and angular manipulation [17]. However, these efforts have been focused on the UV range only, and significant loss in the metal is not negligible for applications from UV to NIR. In order to completely couple light into sub-nanometre-thick graphene by an efficient and cost-effective way, an innovative design of photonic structure from UV to NIR is quite in demand.

In this paper, we propose a simple photonic structure with two unpatterned dielectric layers for complete optical absorption in graphene. Our approach adopts a scheme in combination with optical total reflection and coherent perfect absorption, and this avoids the use of high-lossy materials and complicated nanofabrication for the photonic structure. In order to achieve perfect optical absorption in graphene for various applications, we perform a systematic study on structural design, material selection, angle and polarization of incident light for the UV-NIR range. Our methods demonstrate promising potential of graphene for photonic applications on highperformance devices of photo-detection, photovoltaics, optical communication, photothermal emission, chemical and biomedical sensing.

\section{Theoretical model and research method}


In this study, we adopt a single-channel coherent perfect absorber for complete light absorption in graphene with a thickness of sub-nanometre. The proposed photonic structure consists of two lossless dielectric layers, in combination with graphene, as shown in Fig. 1(a). The refractive index $n_{1}$ and $n_{2}$ for both of the dielectric layers are larger than the air refractive index $n_{3}$, Layer 2 has a thickness of $d_{2}$, and the location of graphene on Boundary A (interface of Layer 1/ Layer 2) or Boundary B (interface of Layer 2/Layer 3) is investigated. The incident light is introduced from Layer 1, and its incident angle and polarization are manipulated with the design of material and structure, in order to achieve perfect optical absorption in graphene. An analytical method based on characteristic matrices of multilayer thin films is used to calculate and optimize the optical absorption in graphene [18]. Full wave numerical simulation using a finite element method is also adopted to confirm the analytical optimization and provide a more comprehensive physical picture [19]. In this study, we mainly focus on graphene with a sub-nanometre thickness, which is much smaller than the wavelength of light and only induces the in-plane carrier transport for optical excitations. A wavelengthdependent surface conductivity $\sigma$ is used to describe the optical response of graphene. The Fano model for the many-body effects and the Kubo formalism for an independent particle are combined to describe the optical conductivity of graphene from UV to NIR $[20,21]$. The Fano model for the excitation of high optical energy can be expressed as below,

$$
\begin{aligned}
\sigma_{\text {Fano }}(\lambda) & =\frac{\sigma_{C B}(\lambda) \cdot(q+\varepsilon)^{2}}{1+\varepsilon^{2}} \\
\varepsilon & =\frac{h c / \lambda-E_{r}}{\Gamma_{r} / 2}
\end{aligned}
$$

where $\lambda, h$ and $c$ denote the free space wavelength, Plank constant, speed of light respectively; $\varepsilon$ is the normalized energy by width $\Gamma_{r}=0.78 \mathrm{eV}$ relative to the resonance energy $E_{r}=5.02 \mathrm{eV}$ of the perturbed exciton; $\sigma_{C B}(\lambda)$ is the continuum background obtained by the calculation for a many-body system. The NIR conductivity of plural form by the Kubo formalism can be expressed by the following equations,

$$
\begin{gathered}
\sigma\left(\omega, \mu_{c}, \Gamma, T\right)=\sigma_{\text {int } r a}+\sigma_{\text {inter }} \\
\sigma_{\text {intra }}=\frac{j e^{2}}{\pi \hbar^{2}(\omega-j 2 \Gamma)} \int_{0}^{\infty} \xi\left(\frac{\partial f_{d}\left(\xi, \mu_{c}, T\right)}{\partial \xi}-\frac{\partial f_{d}\left(-\xi, \mu_{c}, T\right)}{\partial \xi}\right) d \xi
\end{gathered}
$$




$$
\begin{gathered}
\sigma_{\text {inter }}=-\frac{j e^{2}(\omega-j 2 \Gamma)}{\pi \hbar^{2}} \int_{0}^{\infty} \frac{f_{d}\left(-\xi, \mu_{c}, T\right)-f_{d}\left(\xi, \mu_{c}, T\right)}{(\omega-j 2 \Gamma)^{2}-4(\xi / \hbar)^{2}} d \xi \\
f_{d}\left(\xi, \mu_{c}, T\right)=\left(e^{\left(\xi-\mu_{c}\right) / k_{B} T}+1\right)^{-1}
\end{gathered}
$$

Here, $\sigma_{\text {intra }}$ and $\sigma_{\text {intra }}$ represent the optical conductivity contributions from the intraband and interband transitions, respectively. In the equations, $\hbar, k_{B}$, and $e$ denote the reduced Plank constant, Boltzmann constant, and electron charge, respectively. $T, \xi, \mu_{c}$ and $\omega$ represent Kelvin temperature, electron energy, chemistry potential and angular frequency, respectively. $\Gamma$ is scattering rate and is taken as $16.67 p s^{-1} \cdot f_{d}\left(\xi, \mu_{c}, T\right)$ is the Fermi-Dirac distribution function. The wavelength-dependent conductivity of graphene from UV to NIR is shown in Fig. 1(b). Based on the optical conductivity, graphene monolayer can also be considered as a lossy dielectric material with a thickness $d_{g}$ of $0.35 \mathrm{~nm}[22]$.

(a)

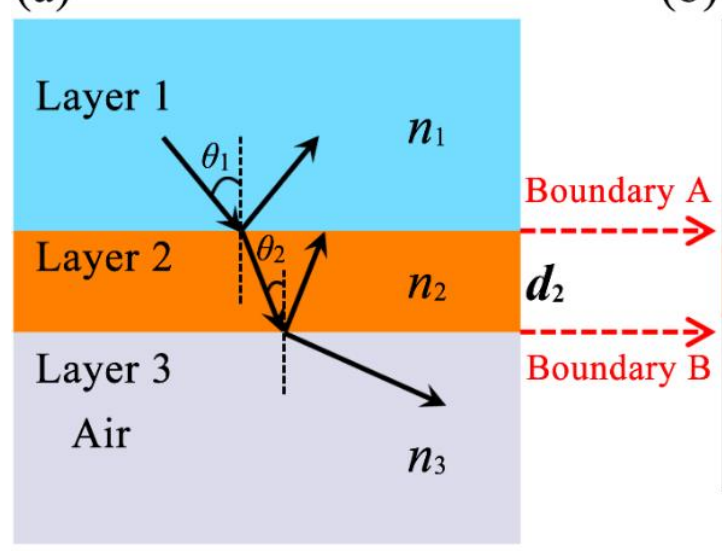

(b)

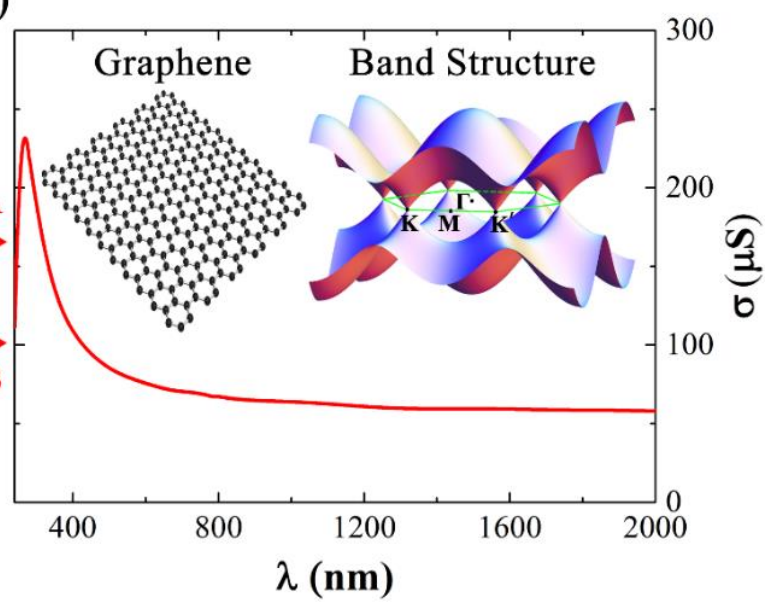

Fig. 1. (a) Schematic drawing of the proposed photonic structure with two dielectric layers, including Layer 1 and Layer 2, where $n_{1}, n_{2}$ and $n_{3}$ represent the corresponding refractive index. (b) Optical conductivity of graphene from $240 \mathrm{~nm}$ to $2000 \mathrm{~nm}$, and insets are the atomic structure and band structure of graphene, where the symbol $\mathrm{M}$ denotes the saddle point singularity.

The proposed photonic structure can be assumed as a two-port optical network with both a dielectric channel (Layer 1) and an air channel (Layer 3). The relationship between input and output waves is described by the following equation,

$$
\left(\begin{array}{l}
b_{1} \\
b_{3}
\end{array}\right)=\hat{S}\left(\begin{array}{l}
a_{1} \\
a_{3}
\end{array}\right), \hat{S}=\left(\begin{array}{ll}
r_{11} & t_{13} \\
t_{31} & r_{33}
\end{array}\right)
$$


Here, the complex scalars $a_{i}$ and $b_{i}$ represent the input and output wave amplitudes in the $i$-th layer, respectively, as shown in Fig. 1(a); $r_{i i}$ are the reflection coefficients and $t_{i j}$ are the transmission coefficients. The scattering matrix $\hat{S}$ is derived from Maxwell's equations by using the method of characteristic matrices, and it depends on the material optical constants, the thickness of Layer 2, the operating wavelength, the light incident angle and polarization. When the photonic structure is fixed, one can further functionalize its propagation properties by tuning light incident angle and polarization. Given the optical incident angle in Layer 1, the light angle through all remaining layers can be calculated using Snell's Law of Refraction $N_{1} \sin \theta_{1}=N_{i} \sin \theta_{i}$, where $N_{i}=n_{i}+k_{i}$ is the complex refractive index of Layer $i, k_{i}$ equals to zero for the lossless layer $i, \theta_{i}$ is the light angle for layer $i$, and $\theta_{i}$ can be complex-valued to properly allow for the effects of attenuation in lossy medium. Since the proposed network has only one input light excitation from the dielectric channel in Layer 1, we only need considering the output wave components $t_{31}$ and $r_{11}$ to be zero for perfect absorption occurring in the network.

Primarily, we investigate the condition of blocking transmission from Layer 1 to Layer 3 (i.e. $t_{31}=0$ ). In view of the theory of coherent perfect absorption [23], when graphene is in between Layer 1 and Layer 3, only one light input by normal incidence from Layer 1 cannot block all the transmission, because graphene is extremely thin even though it is optical lossy. Thus, a scheme of tuning the incident angle for the multilayer structure might be used to eliminate the transmission. In order to simplify this investigation, we start from the study on the optical propagation properties of the proposed structure without graphene in view of the change of incident angle, as shown in Fig. 2. There is a critical angle $\theta_{c}$ of total reflection for the entire photonic structure, and the value of this angle is only determined by the refractive index difference between Layer 1 and Layer 3, which is to be further proved by the following demonstration. First, we get the equation $n_{2} \sin \theta_{2}=n_{3} \sin \theta_{3}$ based on Snell's Law, calculate the critical angle $\theta_{2 c}$ for total optical reflection on Boundary B from Layer 2 to Layer 3, and obtain the equation $\sin \theta_{2 c}=n_{3} / n_{2}$. Second, the light angle $\theta_{2 c}$ in Layer 2 corresponds to the specific incident angle $\theta_{1}$ in Layer 1 , and we have $\theta_{1}=\arcsin \left(n_{3} / n_{1}\right)$, which is calculated from $n_{1} \sin \theta_{1}=n_{2} \sin \theta_{2 c}$ by using Snell's Law on Boundary A. Third, we consider the condition for maximum incident angle $\theta_{1 m}$ for light transmission through Boundary A from Layer 1 to Layer 2. If $n_{1}<n_{2}$, there is no total reflection on Boundary A from Layer 1 to Layer 2, the maximum incident angle $\theta_{1 m}$ allowing for transmission is up to $90^{\circ}$. If $n_{1} \geq n_{2}, \theta_{1 m}$ is determined by the critical angle $\theta_{1 c}=\arcsin \left(n_{2} / n_{1}\right)$ for total reflection from Layer 1 to Layer 2. Therefore, we obtain the following comparison expression, 


$$
\theta_{1}=\arcsin \left(n_{3} / n_{1}\right)<\theta_{1 m}= \begin{cases}\arcsin \left(n_{2} / n_{1}\right), & \text { if } n_{1} \geq n_{2} \\ 90^{\circ} & \text { if } n_{1}<n_{2}\end{cases}
$$

Hence, the critical angle of total optical reflection for the proposed photonic structure is $\theta_{c}=\theta_{1}=\arcsin \left(n_{3} / n_{1}\right)$, which is only determined by the refractive indexes $n_{1}$ and $n_{3}$, regardless of the refractive index and thickness of Layer 2. This propagation property is independent from light polarization, as shown in Fig. 2(a) and Fig. 2(b), where $n_{1}=1.46$, $n_{2}=1.77, n_{3}=1$, and $\theta_{c}=\arcsin \left(n_{3} / n_{1}\right)=43.2^{\circ}$. On the condition for an incident angle $\theta$ smaller than $43.2^{\circ}$, light can be transmitted from Layer 1 to Layer 3, and the photonic structure is a two-port system with incidence, reflectance and transmittance. As observed in Fig. 2(a) and Fig. 2(b), when the incident angle $\theta$ is larger than $43.2^{\circ}$, light transmission from Layer 1 to Layer 3 is totally blocked (i.e. $t_{31}=0$ ), the bottom surface of Layer 2 plays a role of perfect reflecting mirror, and the optical network acts as a one-port system with only incidence and reflectance. Furthermore, when the graphene monolayer is introduced to the location of Boundary A or Boundary B as shown in Fig. 1 , it does not change the critical angle $\theta_{c}$ of total reflection for the entire structure under both $s$ - and $p$-polarizations (see Fig. S1). This is because graphene can be considered as an extremely thin intermediate layer of lossy dielectric material between Layer 1 and Layer 3, and it doesn't change $\theta_{c}$ determined by $n_{1}$ and $n_{3}$.
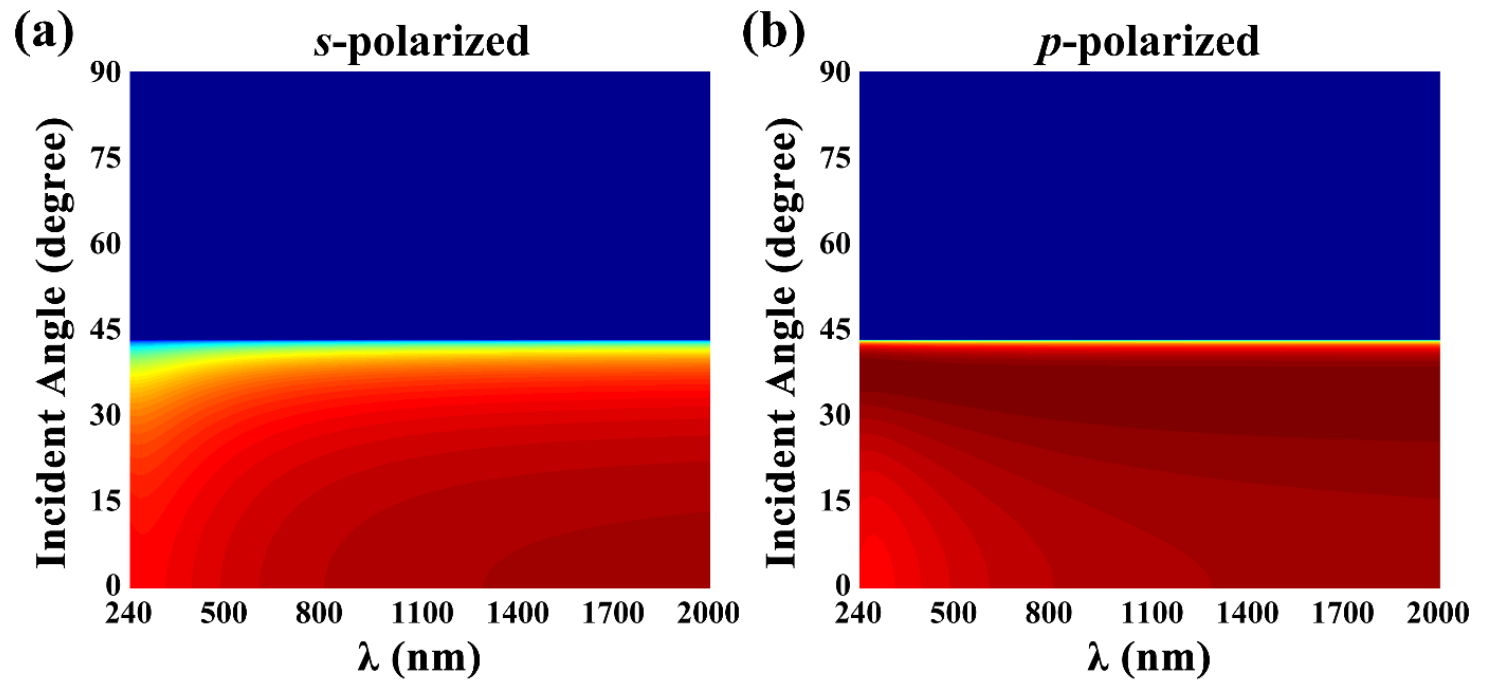

Fig. 2. Optical transmittance of the dielectric/dielectric/air layers, as a function of wavelength and incident angle, where $n_{1}=1.46, n_{2}=1.77, n_{3}=1, d_{2}=40 \mathrm{~nm}$. (a) Incidence of $s$-polarized light. (b) Incidence of $p$-polarized light.

Next, based on the condition of incident angles larger than $\theta_{c}$ for $t_{31}=0$, we discuss how to make the reflection coefficient $r_{11}$ equal to zero for perfect absorbance $(A=1$ - 
$\left|t_{31}\right|^{2}-\left|r_{11}\right|^{2}=1$ ) in the proposed structure with graphene in Boundary A or Boundary B. The layers of dielectric and air are assumed to be lossless, so, all the optical dissipation is attributed to the loss in graphene if the perfect absorption (i.e. $r_{11}=0$ ) is possible. In this study, graphene is assumed as a two-dimensional lossy film with surface optical conductivity and vanishing thickness, which slightly influences the value of $\theta_{c}$ for total reflection on the entire structure. The local optical absorption is induced by the in-plane Ohmic dissipation on the graphene surface, and it is determined by the wavelengthdependant conductivity and the local electric field component parallel to the graphene surface, as shown below,

$$
A(\lambda)=\frac{\sigma(\lambda)}{2}\left|E_{p}(\lambda)\right|^{2}
$$

where $E_{p}$ denotes the parallel component of wavelength-dependant electric field on the graphene surface. When the incident angle is larger than $\theta_{\mathrm{c}}$, the electric field of $p$ polarized light is not parallel to the graphene surface, but the electric field of $s$-polarized light is always parallel to the graphene surface. Hence, only s-polarized light has the possibility to achieve absolute optical absorption based on equation (9), and we only need to focus on the discussions of s-polarized incidence for the next investigations. The tilted optical admittance in Layer $i$ for $s$-polarized light with an angle $\theta_{i}$ can be expressed as $\eta_{i}=H_{i} \cos \theta_{i} / E_{i}$, where $H_{i}$ and $E_{i}$ represent the magnetic field and electric field in Layer $i$, respectively. The phase factor of the positive-going wave in Layer 2 is denoted as $\delta_{2}=2 \pi n_{2} d_{2} \cos \theta_{2} / \lambda$, and the phase factor in graphene is expressed as $\delta_{g}=(2 \pi / \lambda) d_{g}\left(n_{g}^{2}-k_{g}^{2}-n_{1}^{2} \sin ^{2} \theta_{1}-2 j n_{g} k_{g}\right)^{1 / 2}$, where $N_{g}=n_{g}+k_{g}$ is the effective complex refractive index of graphene. The characteristic matrix of Layer 2 and the characteristic matrix of graphene are expressed as below,

$$
\begin{gathered}
M_{2}=\left[\begin{array}{cc}
\cos \delta_{2} & i \sin \delta_{2} / \eta_{2} \\
i \eta_{2} \sin \delta_{2} & \cos \delta_{2}
\end{array}\right] \\
M_{g}=\left[\begin{array}{cc}
\cos \delta_{g} & i \sin \delta_{g} / \eta_{g} \\
i \eta_{g} \sin \delta_{g} & \cos \delta_{g}
\end{array}\right]
\end{gathered}
$$

and the reflection coefficient of the propose structure is shown as below,

$$
r=\frac{\eta_{1}-Y}{\eta_{1}+Y}
$$

where $Y$ represents the titled optical admittance of the assembled material layers except Layer 1. It can be written as below, 


$$
Y= \begin{cases}M_{g} M_{2}\left[\begin{array}{c}
1 \\
\eta_{3}
\end{array}\right], & \text { if graphene is in Boundary A. } \\
M_{2} M_{g}\left[\begin{array}{c}
1 \\
\eta_{3}
\end{array}\right], & \text { if graphene is in Boundary B. }\end{cases}
$$

as observed from equation (10) to equation (13), the reflection coefficient $r$ is a function of the phase thickness of Layer 2, the incident angle and the lossy term of graphene's complex refractive index. In order to achieve perfect optical absorption, the reflectance $R=|r|^{2}$ should equal to zero. Therefore, when all the materials are fixed, the conditions for perfect absorption are mainly determined by the thickness of Layer 2 and the incident angle. Based on equation (8), the condition of $n_{1} \geq n_{2}$ is impossible for equation (12) to get close to a zero value, so we next focus on the condition of $n_{1}<n_{2}$ for perfect absorption in graphene.

\section{Results and discussions}

As shown in Fig. 3(a) and Fig. 3(b), regardless of graphene on Boundary A or Boundary $\mathrm{B}$, the perfect absorbance in monolayer graphene can be reached for a fixed thickness of Layer 2 by using an appropriate coupling angle. As the thickness of Layer 2 increases from $35 \mathrm{~nm}$ to $143 \mathrm{~nm}$, the central wavelengths of absorption bands have a red shift from the UV range $(\lambda=268 \mathrm{~nm}$ and $\lambda=270 \mathrm{~nm}$ for graphene on Boundary A and $\mathrm{B}$, respectively) to the NIR range $(\lambda=1100 \mathrm{~nm}$ and $\lambda=1100 \mathrm{~nm}$ for graphene on Boundary A and B, respectively). Besides, graphene on boundary A (Fig. 3(a)) excites broader absorption bands than graphene on Boundary B (Fig. 3(b)). The analytical results based on the method of characteristic matrices demonstrate good consistency with the results from FEM simulations. The perfect absorbance in graphene is attributed to the coherence of the light from the upper and lower interfaces of Layer 2. Thus, the proposed structure with graphene can be considered as a single-channel coherent perfect absorber. The coherent perfect absorption is induced by the light wave coupling within Layer 2 under a certain angle, and almost all the light energy dissipates inside graphene for a wavelength determined by the thickness of Layer 2. Graphene on Boundary A has smaller coupling angles and central wavelengths for perfect absorption than graphene on Boundary B, as shown in Fig. 3(a) and Fig. 3(b). Based on equation (9), the optical absorption is determined by the optical conductivity of graphene and the electric field intensity on it. The conductivity of monolayer graphene around $\lambda=268 \mathrm{~nm}$ is about 3.3 times of that at $\lambda=700 \mathrm{~nm}$. In comparison, as observed by the electric field distributions in Fig. 3(c) and Fig. 3(d), on the coupling conditions for coherent perfect absorption, the 
electric field amplitude at $\lambda=700 \mathrm{~nm}$ is about $1.82\left(3.3^{1 / 2}\right)$ times of that at $\lambda=268 \mathrm{~nm}$. These electric field distributions also confirm that the perfect light absorption originates from the enhancement of in-plane electric field on the graphene surface under $s$ polarized light incidence.

(a)

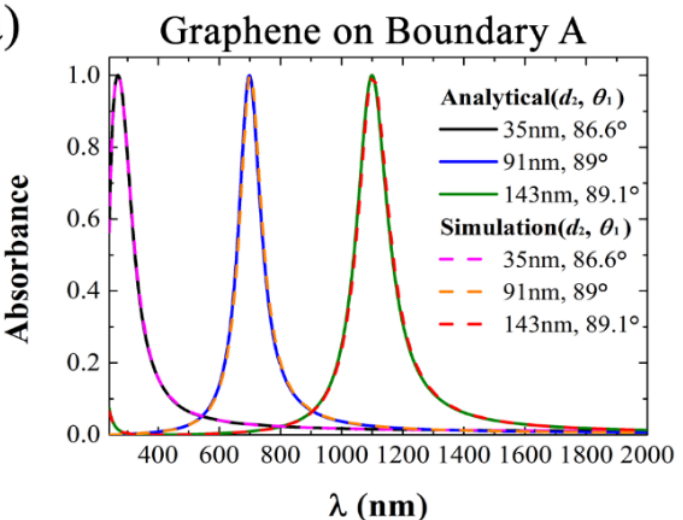

(c)

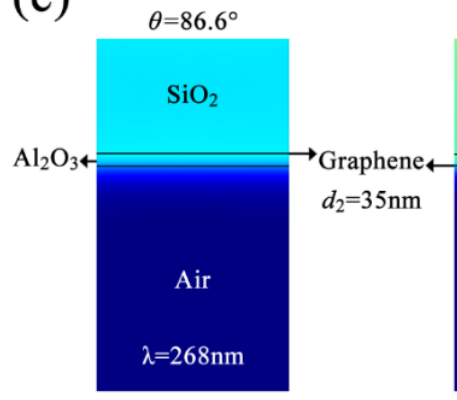

$\theta=88.4^{\circ}$

$\mathrm{SiO}_{2}$

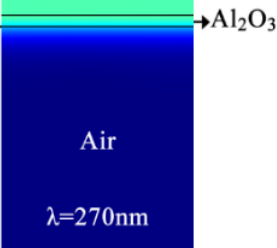

(b)

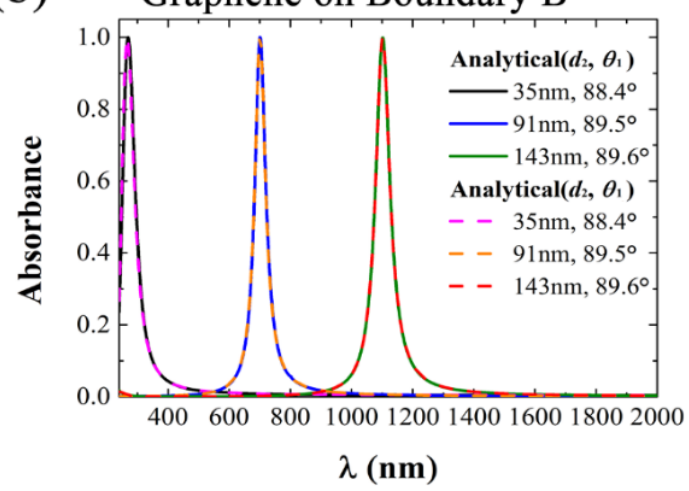

(d)

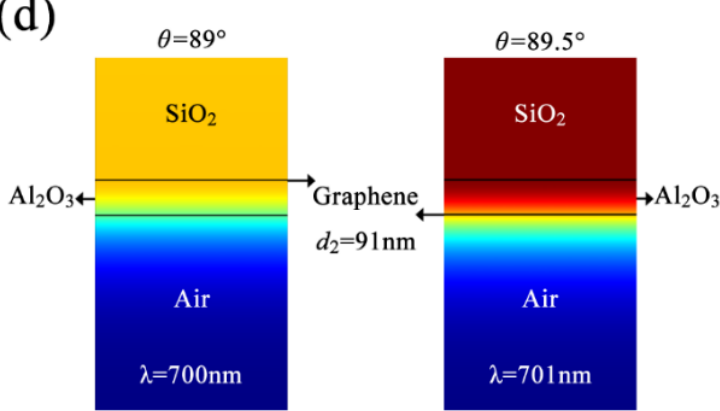

0

$|E|_{\max }$

Fig. 3. (a) Absorbance of graphene on Boundary A for different thicknesses of Layer 2 and corresponding coupling angles. (b) Absorbance of graphene on Boundary A for different thicknesses of Layer 2 and corresponding coupling angles. (c) Electric field distributions for the perfect absorbance, where for $d_{2}=35 \mathrm{~nm}$. (d) Electric field distributions for the perfect absorbance, where for $d_{2}=91 \mathrm{~nm}$. The parameters $n_{1}$ and $n_{2}$ are 1.46 and 1.77 , respectively.

Next, we further discuss the factors that influence the perfect light absorption of graphene. As shown in Fig. 4 (a) and Fig. 4(b), for a fixed thickness of Layer 2, the incident light energy is efficiently coupled into the absorption inside graphene around a certain coupling angle, which is close to $90^{\circ}$, especially for monolayer graphene with a lower optical conductivity. Due to the optical path difference, graphene on Boundary B (Fig. 4(a)) has a smaller angle range for high absorbance compared with graphene on Boundary A (Fig. 4(b)). Despite the absorption bandwidth for wavelengths and incident angles, the locations of graphene on Boundary A and Boundary B have very similar optical response, because the single-channel light coherence is attributed to the optical dissipation on the upper or lower interfaces of Layer 2. For a fixed wavelength, the 
perfect absorption can be engineered and maintained by tuning both the thickness of Layer 2 and the refractive index of Layer 1, as shown in Fig. 4(c). The coherent perfect absorption is determined by the phase factor of Layer 2 as below.

$$
d_{2}=2 \pi n_{2} d_{2} \cos q_{2} / \lambda=(2 \pi / \lambda) d_{2}\left(n_{2}^{2}-n_{1}^{2} \sin ^{2} q_{1}\right)^{1 / 2}
$$

When $\lambda, n_{2}$ and $\theta_{2}$ are fixed, the term $d_{2}\left(n_{2}^{2}-n_{1}^{2} \sin ^{2} q_{1}\right)^{1 / 2}$ should be constant, in order to achieve the perfect absorption based on equations form (10) to (13). So the thickness of Layer 2 increases as the refractive index of Layer 1 becomes large. As observed in Fig. 4(d), the central wavelength of perfect absorption band depends on the thickness of Layer 2, and it linearly increases as the thickness of Layer 2 becomes large, because $\lambda$ is proportional to $d_{2}$ in order to keep $\delta_{2}$ constant for perfect absorption based on equation (14). When the thickness becomes larger, multiple absorption bands appear due to high order coherence modes, as shown in Fig. 4(d). Fig. 5(e) indicates multiple coherence modes with high absorbance ratios can be achieved by increasing the thickness of Layer 2 up to $1 \mu \mathrm{m}$, and the high order modes have narrower absorption bands and smaller operational angles of incidence for high absorbance ratios. Hence, one can tune the thickness of Layer 2 for different absorption bands, multiple absorption bands and operational angle of incidence. Even though Fig. 4(c)-Fig. 4(e) demonstrate the optical properties for graphene on Boundary A only, similar optical properties can be also found for graphene on Boundary B. The angular dependent optical properties of graphene could be applied to implement narrowband perfect absorption, as shown in Fig. 5(f), where a cleaved fibre with alumina and graphene coatings is used to completely absorb the optical signal. Such a design might facilitate the photonic integration in combination with graphene and fibres on many applications for optical communications and optoelectronic devices. 
(a)

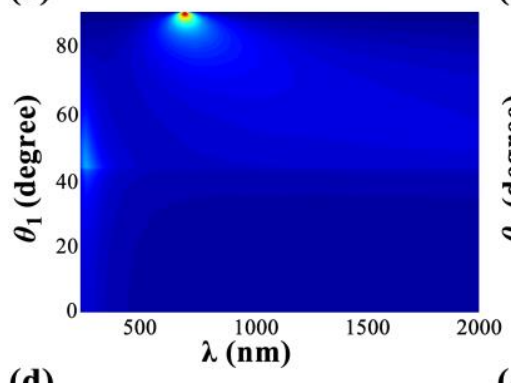

(d)

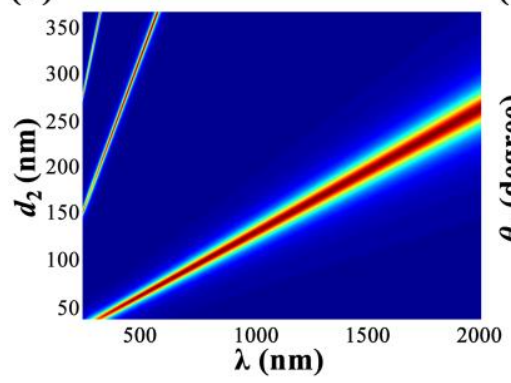

(b)

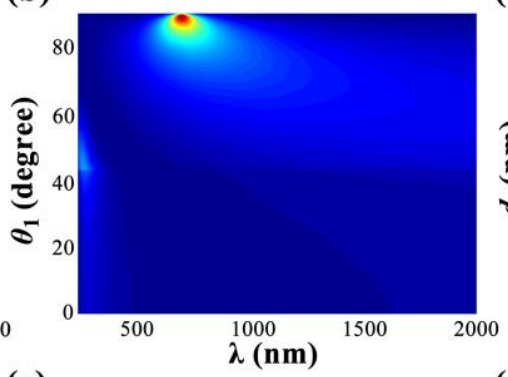

(e)

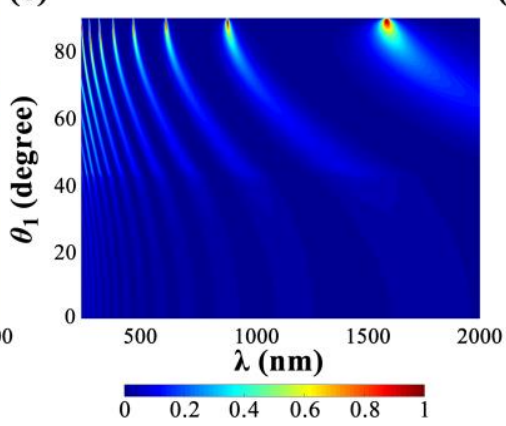

(c)

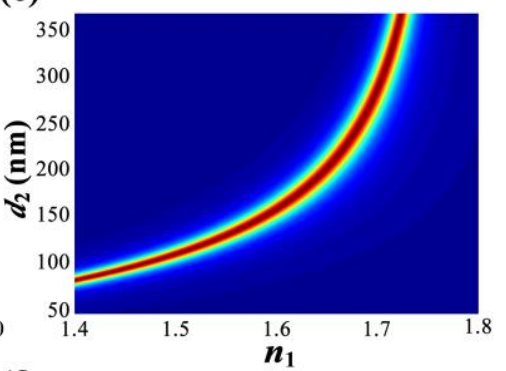

(f)

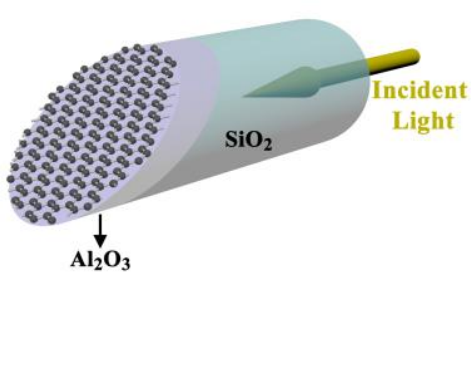

Fig. 4. (a) Absorbance for graphene on Boundary B and (b) absorbance for graphene on Boundary A, as functions of $\lambda$ and incident angle, where $n_{1}=1.46, n_{2}=1.77$ and $d_{2}=91 \mathrm{~nm}$. (c) Absorbance for graphene on Boundary A as a function of $n_{1}$ and $d_{2}$, where $n_{2}=1.77$ and $\lambda=700 \mathrm{~nm}$. (d) Absorbance of graphene on Boundary A as a function of $\lambda$ and $d_{2}$, where $n_{1}=1.46, n_{2}=1.77$ and $\theta_{1}=89^{\circ}$. (e) Absorbance for graphene on Boundary A, as a function of $\lambda$ and $\theta_{1}$, where $n_{1}=1.46, n_{2}=1.77$ and $d_{2}=1 \mu \mathrm{m}$. (f) Cleaved optical fibre with alumina and graphene coatings for perfect light absorption.

The perfect absorption features of the proposed structure can be also adopted for a new optical sensing mechanism. As well known, the surface optical conductivity of graphene is very sensitive to slight environmental contamination, partial chemical bonding or carrier doping [24-27], hence the change of the surface optical conductivity on the 2D material is a critical criterion for sensing applications. As shown in Fig. 5(a), a prism-based structure is used to detect the change of surface optical conductivity on the hexagonal boron nitride $(h \mathrm{BN})$ layer. For instance, localized chemical bonding to the carbon atoms of graphene by oxygen atoms or hydroxyl could reduce the optical conductivity, which can be measured by the reflectance spectra. Such a scheme seems similar to the Kretschmann conFiguration for surface plamon resonance sensing [28], which uses $p$-polarized light to excite surface plamons on a novel metal film. However, our scheme uses s-polarized light and maximizes the interaction between the 2D material and the electric field on the 2D surface by an appropriate coupling angle. The change of optical conductivity due to surface contamination, bonding or doping can be simply detected by measuring the reflectance ratios for a certain wavelength, as shown 
in Fig. 5(b). For a fixed incident angle, the intensity of reflection signal is very sensitive to the change of the surface optical conductivity (from $\sigma$ to $0.05 \sigma$ ), which implies its promising potential for single molecule spectroscopy. This detection allows for the use of visible light, whose detectors are very popular and inexpensive in various sensing platforms. In addition, such detection method is non-destructive and avoids any contact problem by using electrodes for measuring electrical conductivity on the $2 \mathrm{D}$ material [29].

(a)
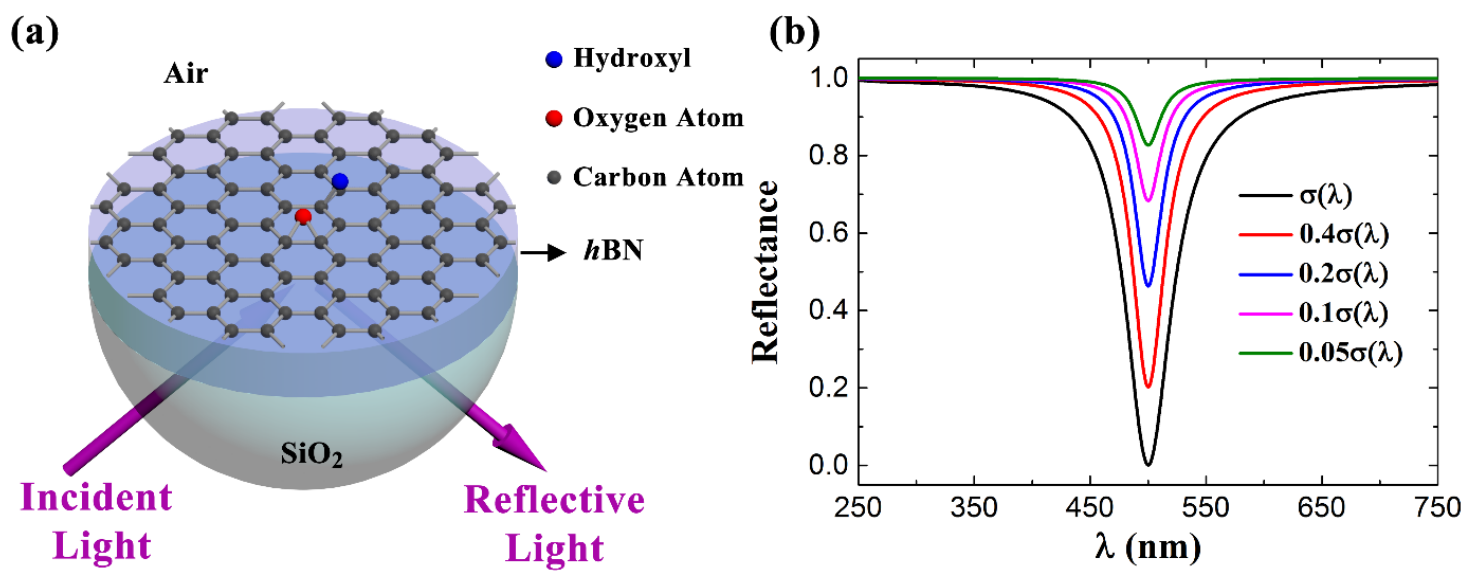

Fig. 5. (a) Optical sensing structure for localized chemical bonding based on graphene, $h \mathrm{BN}$ and a prism. (b) Reflectance spectra as a function of optical conductivity of graphene by using the prism-based structure, where the incident angle and the thickness of $h \mathrm{BN}$ are $89.1^{\circ}$ and $30.9 \mathrm{~nm}$, respectively.

Since the electric-optic modulation of graphene is of great importance in optical communication [30], we next investigate modulation effects of the proposed photonic structure in the NIR range. As show in Fig. 6, the intensity of optical reflectance for graphene on Boundary B or Boundary A is modulated by changing the chemical potential of graphene, which can be implemented by electronic gating on graphene [31]. It is observed in Fig. 6(a) and Fig. 6(c), the optical reflectance ratios around $\lambda=1550 \mathrm{~nm}$ can be tuned by changing the chemical potential $\mu_{c}$ from $0.3 \mathrm{eV}$ to $0.65 \mathrm{eV}$. Around $\lambda=1550 \mathrm{~nm}$, the reflectance of $\mu_{c}=0.3 \mathrm{eV}$ is close to zero due to the effects of coherent perfect absorption, whereas the reflectance of $\mu_{c}=0.65 \mathrm{eV}$ is very large up to $87.6 \%$ and $88 \%$ for graphene on Boundary A and Boundary B, respectively. The reflectance differences are attributed to the significant reduction of optical conductivity based on equation (9). Comparing Fig. 4(a) and Fig. 4(c), we find that graphene on Boundary B has a higher quality factor for the reflectance dip than graphene on Boundary A. In order to evaluate the modulation effects, we define the optical contrast of reflectance by $\left(R_{o n}-R_{\text {off }}\right) / R_{\text {off }}$ [32], where $R_{\text {on }}$ and $R_{\text {off }}$ represent the reflectance for $\mu_{c}=0.65 \mathrm{eV}$ and 
$\mu_{c}=0.3 \mathrm{eV}$, respectively. For graphene on Boundary B (Fig. 6(b)) and Boundary A (Fig. $6(\mathrm{~d})$ ), the optical contrasts around $\lambda=1550 \mathrm{~nm}$ are extremely high, and the modulation efficiency for graphene on Boundary A (9969 at $\lambda=1553 \mathrm{~nm})$ is much higher than that for graphene on Boundary B (1879 at $\lambda=1550 \mathrm{~nm})$. This indicates the promising potential of the proposed photonics structure for developing high-performance electricoptic modulators based on graphene.

(a)

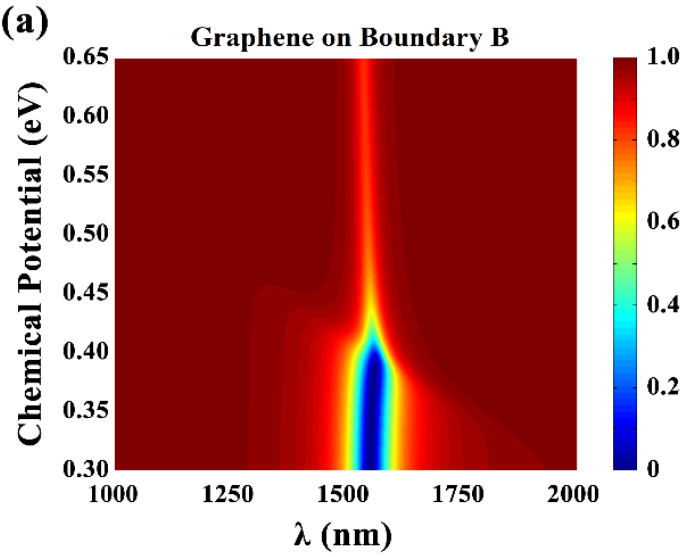

(c)

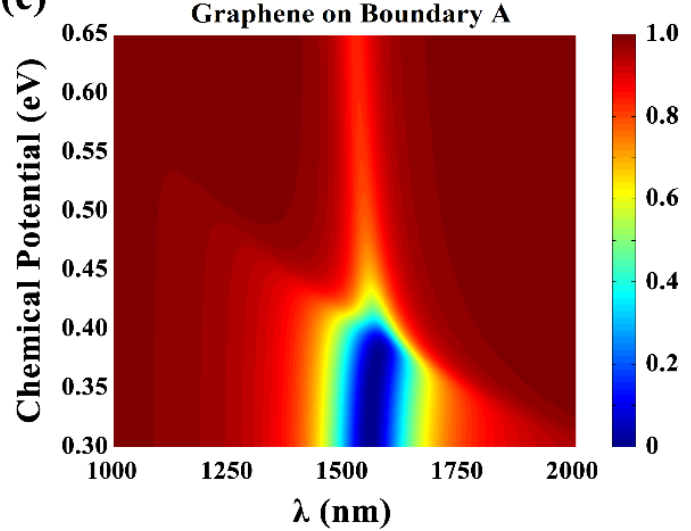

(b)

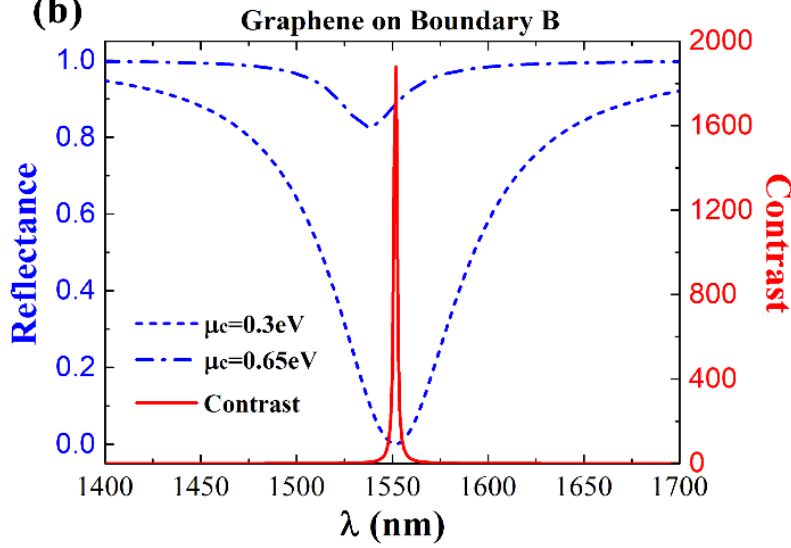

(d)

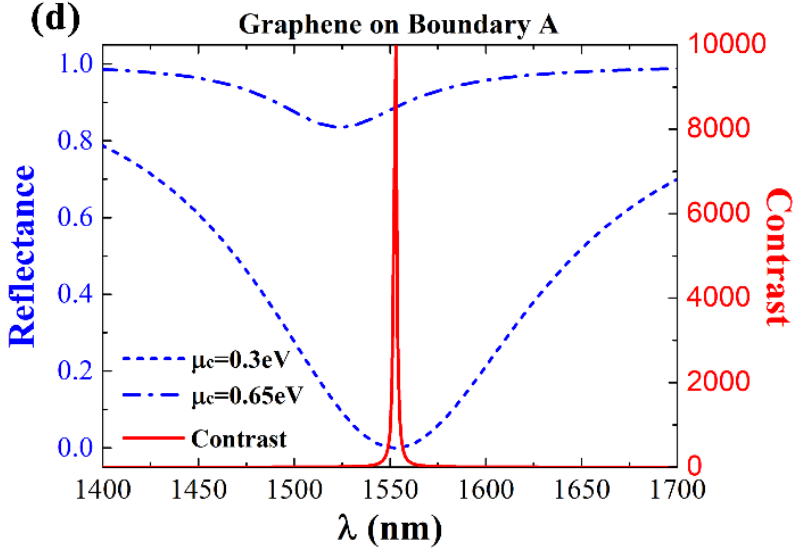

Fig. 6. (a) Reflectance as a function of $\lambda$ and $\mu_{c}$, and (b) reflectance contrast between $\mu_{\mathrm{c}}=0.65 \mathrm{eV}$ and $\mu_{\mathrm{c}}=0.3 \mathrm{eV}$, where graphene is on Boundary $\mathrm{B}$ and $\theta_{1}=89.6^{\circ}$. (c) Reflectance as a function of $\lambda$ and $\mu_{\mathrm{c}}$, and (d) reflectance contrast between $\mu_{\mathrm{c}}=0.65 \mathrm{eV}$ and $\mu_{\mathrm{c}}=0.3 \mathrm{eV}$, where graphene is on Boundary $\mathrm{A}$ and and $\theta_{1}=89.1^{\circ}$. The parameters $n_{1}$, $n_{2}$ and $d_{2}$ are $1.46,1.77$ and $201 \mathrm{~nm}$, respectively.

The above achievements will facilitate the applications on perfect absorption of single-wavelength signal, electric-optic modulation and optical sensing based on graphene, but they depend on the high optical absorption of a narrow band. In order to bring about broadband high absorption, graphene with more atomic layers can be introduced on both Boundary A and Boundary B. As shown in Fig. 5 (a) and Fig. 5(b), broadband high absorbance is obtained in the visible range, when the incident angle is larger than the critical angle of $43.2^{\circ}$. These two Figures also demonstrate that the maximum absorbance ratios above $95 \%$ are focused around $\lambda=520 \mathrm{~nm}$, which is 
determined by the fixed thickness of Layer2. The absorbance above $60 \%$ can be maintained for the wide ranges of wavelengths and incident angles. In contrast, the absorbance ratio is significantly reduced for the incident angle below the critical angle of $43.2^{\circ}$. The broadband optical absorption is of great importance for developing many optical devices based on graphene, such as solar cells, light emmiters and photothermal therapy [33-35]. Based on the modelling theory, this angle can be tuned by changing the ratio of refractive index between Layer 3 and Layer 1. The reconFigurable light response based on angular manipulation might be applied on broadband optical switching and broadband filtering of different angular signals. Compared to graphene with three atomic layers in Fig. 5(a), graphene with four atomic layers in Fig. 5(b) maintains broadband high absorbance for a larger range of incident angle. In this structural conFiguration, Layer 2 is sandwiched by two graphene layers, and it acts as an optical cavity with light energy dissipation on both Boundary A and Boundary B. The use of graphene with more atomic layers reduces the quality factor of the cavity, and induces more broadband optical absorption for more incident angles.
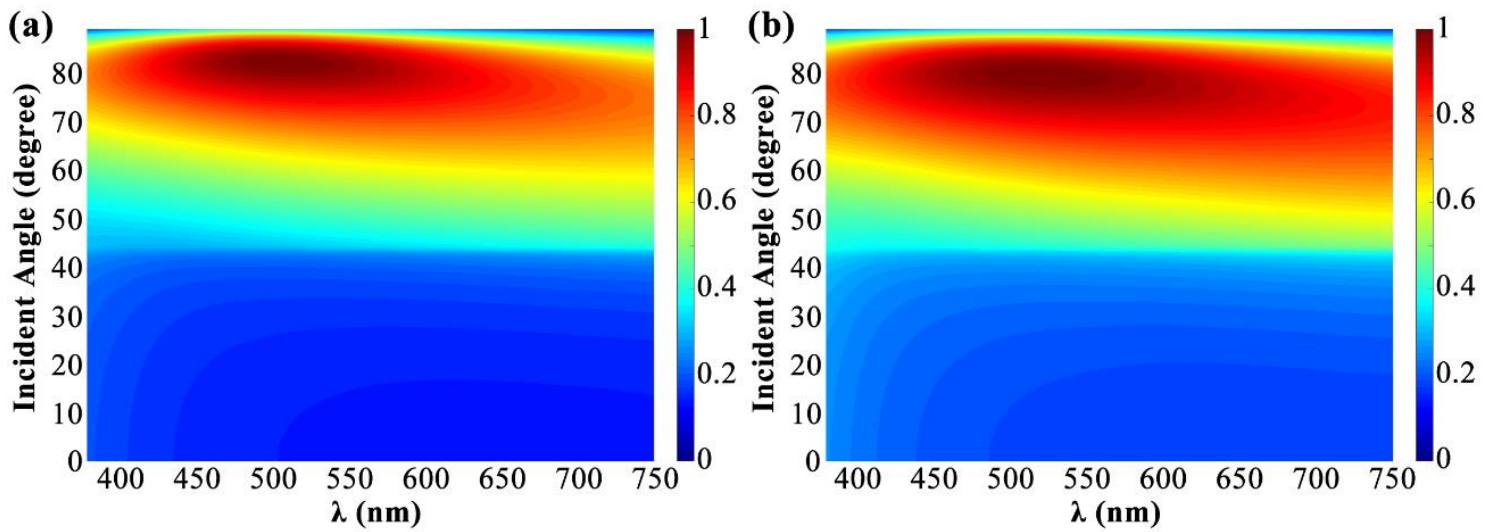

Fig. 7. (a) Absorbance as a function of $\lambda$ and $\theta_{1}$, in which both Boundary $A$ and Boundary B have graphene with three atomic layers. (b) Absorbance as a function of $\lambda$ and $\theta_{1}$, in which both Boundary $\mathrm{A}$ and Boundary $\mathrm{B}$ have graphene with four atomic layers. Here, Layer 1 is silica $\left(\mathrm{n}_{1}=1.46\right)$, Layer 2 is alumina $\left(n_{2}=1.77\right)$, Layer 3 is air $\left(n_{3}=1\right)$, and $d_{2}$ equals to $64.8 \mathrm{~nm}$.

\section{Conclusions}

We have proposed a simple photonic structure with two unpatterned dielectric layers, in order to achieve perfect light absorption in the spectral range from UV to NIR. This structure acts as a single-channel coherent perfect absorber induced by the selfinterference mode, and it avoid the fussy patterning on graphene and the use of complicated coherent light beam systems. The perfect light absorption can be 
implemented by a cleaved optical fibre or optical cable, which will facilitate the combination between graphene and integrated optical systems. The extremely high light-matter interaction and narrowband absorption will enable ultrasensitive sensing and high-performance optical modulation based on graphene. Broadband high optical absorption can also be reached with the effects of angular selectivity. Our research provides a comprehensive physical picture for graphene and its future application in the UV-NIR range, and also paves the way for various new applications based on graphene in the optics and optoelectronics community.

\section{Acknowledgements}

This work was supported by China Scholarship Council (201706315004), Fujian Provincial Department of Science and Technology (2017J01123), and National Natural Science Foundation of China (61307042).

\section{References}

[1] F. Xia, H. Wang, D. Xiao, M. Dubey, A. Ramasubramaniam, Two-dimensional material nanophotonics, Nat. Photonics 2014; 8 (12): 899.

[2] K.S. Novoselov, A. Mishchenko, A. Carvalho, A.H.C. Neto, 2D materials and van der Waals heterostructures, Science 2016; 353 (6298): aac9439.

[3] Z. Zhang, P. Chen, X. Duan, K. Zang, J. Luo, X. Duan, Robust epitaxial growth of two-dimensional heterostructures, multiheterostructures, and superlattices, Science 2017; 357 (6353): 788-92.

[4] P.K. Sahoo, S. Memaran, Y. Xin, L. Balicas, H.R. Gutiérrez, One-pot growth of two-dimensional lateral heterostructures via sequential edge-epitaxy, Nature 2018; 553 (7686): 63.

[5] K.F. Mak, J. Shan, Photonics and optoelectronics of 2D semiconductor transition metal dichalcogenides, Nat. Photonics 2016; 10 (4): 216.

[6] H.-L. Liu, S. Siregar, E.H. Hasdeo, Y. Kumamoto, C.-C. Shen, C.-C. Cheng et al., Deep-ultraviolet Raman scattering studies of monolayer graphene thin films, Carbon 2015; 81: 807-13.

[7] D. Rodrigo, O. Limaj, D. Janner, D. Etezadi, F.J.G. de Abajo, V. Pruneri et al., Midinfrared plasmonic biosensing with graphene, Science 2015; 349 (6244): 165-8.

[8] Z. Sun, A. Martinez, F. Wang, Optical modulators with 2D layered materials, Nat. Photonics 2016; 10 (4): 227-38.

[9] K.F. Mak, L. Ju, F. Wang, T.F. Heinz, Optical spectroscopy of graphene: from the far infrared to the ultraviolet, Solid State Commun 2012; 152 (15): 1341-9. 
[10] L. Ye, Y. Chen, G. Cai, N. Liu, J. Zhu, Z. Song et al., Broadband absorber with periodically sinusoidally-patterned graphene layer in terahertz range, Opt. Express 2017; 25 (10): 11223-32.

[11] T. Low, P. Avouris, Graphene plasmonics for terahertz to mid-infrared applications, ACS Nano 2014; 8 (2): 1086-101.

[12] R.R. Nair, P. Blake, A.N. Grigorenko, K.S. Novoselov, T.J. Booth, T. Stauber et al., Fine structure constant defines visual transparency of graphene, Science 2008; 320 (5881): 1308 .

[13] M. Liu, X. Yin, E. Ulin-Avila, B. Geng, T. Zentgraf, L. Ju et al., A graphene-based broadband optical modulator, Nature 2011; 474 (7349): 64.

[14] J.R. Piper, S. Fan, Total absorption in a graphene monolayer in the optical regime by critical coupling with a photonic crystal guided resonance, Acs Photonics 2014; 1 (4): 347-53.

[15] Y. Cai, J. Zhu, Q.H. Liu, Tunable enhanced optical absorption of graphene using plasmonic perfect absorbers, Appl. Phys. Lett 2015; 106 (4): 43105.

[16] M. Furchi, A. Urich, A. Pospischil, G. Lilley, K. Unterrainer, H. Detz et al., Microcavity-integrated graphene photodetector, Nano Lett 2012; 12 (6): 2773-7.

[17] J. Zhu, S. Yan, N. Feng, L. Ye, J.-Y. Ou, Q.H. Liu, Near unity ultraviolet absorption in graphene without patterning, Appl. Phys. Lett 2018; 112 (15): 153106.

[18] H.A. Macleod, H.A. Macleod, Thin-film optical filters. CRC Press; 2010.

[19] J. Niu, M. Luo, J. Zhu, Q.H. Liu, Enhanced plasmonic light absorption engineering of graphene: simulation by boundary-integral spectral element method, Opt. Express 2015; 23 (4): 4539-51.

[20] K.F. Mak, J. Shan, T.F. Heinz, Seeing many-body effects in single-and few-layer graphene: observation of two-dimensional saddle-point excitons, Phys. Rev. Lett 2011; 106 (4): 46401.

[21] Y. Cai, J. Zhu, Q.H. Liu, T. Lin, J. Zhou, L. Ye et al., Enhanced spatial nearinfrared modulation of graphene-loaded perfect absorbers using plasmonic nanoslits, Opt. Express 2015; 23 (25): 32318-28.

[22] J. Zhu, J. Cheng, L. Zhang, Q.H. Liu, Modeling of 2D graphene material for plasmonic hybrid waveguide with enhancedz near-infrared modulation, Mater. Lett 2016; 186: 53-6.

[23] D.G. Baranov, A. Krasnok, T. Shegai, A. Alù, Y. Chong, Coherent perfect absorbers: linear control of light with light, Nat. Rev. Mat 2017; 2 (12): 17064. 
[24] V. Georgakilas, J.N. Tiwari, K.C. Kemp, J.A. Perman, A.B. Bourlinos, K.S. Kim et al., Noncovalent Functionalization of Graphene and Graphene Oxide for Energy Materials, Biosensing, Catalytic, and Biomedical Applications, Chem. Rev 2016; 116 (9): 5464.

[25] K.P. Loh, Q. Bao, G. Eda, M. Chhowalla, Graphene oxide as a chemically tunable platform for optical applications, Nat. Chem 2010; 2(12): 1015-24.

[26] K. Berke, S. Tongay, M. Lemaitre, Z. Nasrollahi, D. Tanner, B. Appleton et al., Stable hole doping of graphene for low electrical resistance and high optical transparency, Nanotechnology 2011; 22 (42): 425701.

[27] M. Houmad, H. Zaari, A. Benyoussef, A. El Kenz, H. Ez-Zahraouy, Optical conductivity enhancement and band gap opening with silicon doped graphene, Carbon 2015; 94:1021-7.

[28] I.D. Villar, V. Torres, M. Beruete, Experimental demonstration of lossy mode and surface plasmon resonance generation with Kretschmann conFiguration, Opt. Lett 2015; 40 (20): 4739.

[29] A. Gahoi, S. Wagner, A. Bablich, S. Kataria, V. Passi, M.C. Lemme, Contact resistance study of various metal electrodes with CVD graphene, Solid. State. Electron 2016; 125: 234-9.

[30] Y. Ding, X. Zhu, S. Xiao, H. Hu, L.H. Frandsen, N.A. Mortensen et al., Effective Electro-Optical Modulation with High Extinction Ratio by a Graphene-Silicon Microring Resonator, Nano Lett 2015; 15 (7): 4393-400.

[31] W. Zhao, Z. Lu, Nanoscale electro-optic modulators based on graphene-slot waveguides, J. Opt. Soc. Am. B Opt. Phys 2012; 29 (6): 1490-6.

[32] J.Y. Ou, E. Plum, J. Zhang, N.I. Zheludev, An electromechanically reconFigurable plasmonic metamaterial operating in the near-infrared., Nat. Nanotechnol 2013; 8 (4): 252-5.

[33] X. Miao, S. Tongay, M.K. Petterson, K. Berke, A.G. Rinzler, B.R. Appleton et al., High efficiency graphene solar cells by chemical doping., Nano Lett 2012; 12 (6): 2745. [34] J. Sloan, N. Rivera, M. Soljačić, I. Kaminer, Tunable UV-Emitters through Graphene Plasmonics, Nano Lett 2017; 18 (1): 308-13.

[35] O. Akhavan, E. Ghaderi, Graphene nanomesh promises extremely efficient in vivo photothermal therapy, Small 2013; 9 (21): 3593-601. 